Manuelle Medizin 2010 • 48:81-82

DOI 10.1007/s00337-010-0742-6

(c) Springer-Verlag 2010

\author{
W. Coenen \\ Villingen-Schwenningen
}

\title{
Manuelle Medizin bei Kindern - ein Sprössling mit Zukunft
}

geht. Diese entwicklungsneurologische Indikation umfasst die Folgen funktioneller und/oder struktureller Störungen des Bewegungssystems und des zentralen Nervensystems mit den unterschiedlichsten und altersabhängigen Symptombildern. Das erfordert neue diagnostische Konzepte und angepasste, kindgerechte manualmedizinische Behandlungstechniken. Die frühzeitige Erkennung sensomotorischer Störungen unterschiedlicher Herkunft und eine frühzeitig einsetzende Behandlung dürften neben den medizinischen und humanitären Gesichtspunkten auch von volkswirtschaftlicher Bedeutung sein, da in vielen Fällen aufwendige Förderungsprogramme im weiteren Verlauf vermeidbar sind. Die manuelle Medizin bei Kindern kann dazu einen wichtigen Beitrag leisten.

\section{Schmerzen und Marotten}

Kinder sind keine kleinen Erwachsenen. Diese Binsenweisheit findet in der täglichen Praxis ihre Bestätigung: Kinder haben eine andere Denkweise, ein anderes Körpergefühl und eine andere Wahrnehmung ihrer Umwelt als Erwachsene. Die Beurteilung von Schmerzzuständen oder Bewegungsauffälligkeiten, die oft ohne erkennbaren Anlass auftreten, erfordert bei Kindern daher eine der Mentalität und dem Alter angepasste Untersuchungsstrategie. Schon manches als Marotte gedeutete Hinken bei Kleinkindern, mancher sog. Wachstumsschmerz oder eine Coxitis fugax ohne nachweisbaren Gelenkerguss entpuppte sich als Ausdruck einer segmentalen Dysfunkti- on der Lendenwirbelsäule oder des Sakroiliakalgelenkes; auch ein rezidivierender Schreibkrampf lässt sich nicht selten mit einer manuellen Behandlung der Halswirbelsäule beheben, um nur einige Beispiele zu nennen. Zunehmend tritt auch die Bedeutung kraniomandibulärer Dysfunktionen bei verschiedenen Störungsbildern im Wachstumsverlauf hervor, nicht zuletzt auch im Zusammenhang mit Pathogenese und Progredienz von Adoleszentenskoliosen.

Seit Beginn der 1990er Jahre sind in dieser Zeitschrift zahlreiche Publikationen zur manuellen Medizin bei Kindern erschienen, wobei es lange Zeit meist dieselben Autoren waren, die zum Thema beitrugen. Auf Veranlassung der DGMM wurde 1992 ein Arbeitskreis manuelle Medizin bei Kindern etabliert, der Fragen der Pathologie, Diagnostik und Therapie zu bestimmten Schwerpunktthemen erörtert: infantile Zerebralparese, Tonusasymmetriesyndrom, sensomotorische Dyskybernese, Hüftdysplasie, Skoliosen, kindliche Schmerzsyndrome und neuromuskuläre Erkrankungen. Vier Jahre später erstellte die Manuelle Medizin ein Themenheft mit ersten Leitlinien zur manuellen Medizin bei Kindern als Ergebnis der Tätigkeit des Arbeitskreises.

Im vorliegenden Heft sind kindertherapeutische Beiträge zu unterschiedlichen Themen zusammengestellt, in denen Möglichkeiten und Grenzen der manuellen Medizin bei Kindern diskutiert werden. Weitere Arbeiten zu diesem Thema sollen in nachfolgenden Ausgaben erscheinen. 


\section{Mauerblümchen}

Gemessen an der Gesamtzahl der Ärztinnen und Ärzte mit der Zusatzbezeichnung „Manuelle Medizin“ ist die Schar derjenigen, die sich systematisch mit kindertherapeutischen Themen aus manualmedizinischer Sicht beschäftigen, nicht sonderlich groß, obwohl die in der DGMM vertretenen Gesellschaften entsprechende Fortbildungskurse im Programm haben. Allerdings scheint das Interesse an der manuellen Medizin bei Kindern in den letzten Jahren zu steigen.

In der Ärztegesellschaft für Manuelle Medizin bei Kindern und Atlastherapie (ÄMKA, früher ÄGAMK) finden Kolleginnen und Kollegen aus allen drei Schulen der DGMM zum Erfahrungsaustausch zusammen. Die ÄMKA bietet seit Anfang der 1990er Jahre Ausbildungskurse in manueller Medizin bei Kindern an und lehrt ein standardisiertes Untersuchungs- und Behandlungskonzept für Säuglinge und Kinder. Das Hauptgewicht liegt dabei auf der Erkennung und Behandlung frühkindlicher sensomotorischer Störungen, kindlicher Schmerzsyndrome und funktioneller Störungen bei Hüftdysplasie, Skoliose und posttraumatischen Zuständen. Den Kern des therapeutischen Konzeptes bilden Manipulationstechniken, osteopathische Behandlungsformen und nicht zuletzt die Atlastherapie nach Arlen.

Manuelle Medizin bei Kindern war längere Zeit ein Mauerblümchen im Schatten der manualmedizinischen Schmerztherapie und Rehabilitationsmedizin. Sie hat sich aber inzwischen zu einem kräftigen Sprössling entwickelt, dessen Zukunft vor allem in der entwicklungsneurologischen Indikation liegt: der Erkennung und Behandlung zerebral und nichtzerebral bedingter sensomotorischer Störungen samt deren Begleitsymptome. Hier übertrifft die manuelle Medizin vielfach die Möglichkeiten der etablierten physiotherapeutischen und ergotherapeutischen Konzepte.

In einer Zeit grundlegender Änderungen der gesundheitspolitischen Strukturen bietet sich dem Manualmediziner mit der Hinwendung zur manuellen Medizin bei Kindern eine neue Perspektive zur Gestaltung seiner ärztlichen Tätigkeit. Diese Chance gilt es zu nutzen, bevor an- dere, auch nichtärztliche Gruppierung das Gebiet für sich beanspruchen.

\section{Mit besten Grüßen}

Ihr

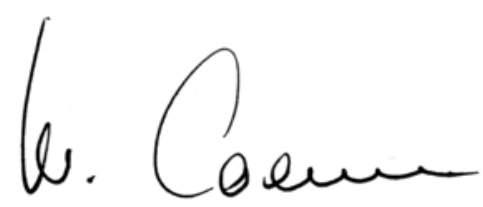

Wilfrid Coenen

\section{Korrespondenzadresse \\ Dr.W. Coenen \\ Schäfersteig 29 \\ 78048 Villingen-Schwenningen \\ drcoenenw@aol.com}

\section{Kein Schmerzmittel vor sport- lichen Höchstleistungen}

Fast zwei Drittel der Läufer beim letztjährigen Bonn-Marathon hatten vor dem Start Schmerzmittel eingenommen, meistens aus pharmakologischer Sicht falsche Präparate oder falsche Dosen. Das ergab eine Umfrage unter 1.000 Marathoni. Die Forscher warnen Sportler eindringlich vor solchem Leichtsinn: Studien zeigen, dass die Wirkstoffe die durch sportliche Anstrengung ohnehin verursachten Belastungen für Magen-Darm-Trakt, Niere und Kreislauf verstärken. Die Spezialisten raten dazu, wenn überhaupt, erst nach der sportlichen Aktivität Schmerzmittel einzunehmen und mit bestehenden Schmerzen gar nicht erst zu starten.

Das unnötige Trinken großer Mengen Mineralwasser während des Laufens erhöht außerdem die Gefährdung des Herz-Kreislauf-Systems. Weniger Wasser mit mehr Kochsalz (1g/l) wäre besser als Magnesiumtabletten in (normalem) Mineralwasser, da diese das Auftreten von Durchfällen begünstigen, ohne Krämpfe sicher zu verhindern.

Quelle: Deutsche Gesellschaft zum Studium des Schmerzes e.V. (DGSS), www. dgss.org 